\title{
Z-open sets in a Neutrosophic Topological Spaces
}

\author{
N. Moogambigai ${ }^{11}$, A. Vadivel ${ }^{2 \dagger}$ and S. Tamilselvan ${ }^{32}$ \\ Corresponding author: A. Vadivel \\ December 13, 2020
}

Article History: Received: 11 January 2021; Accepted: 27 February 2021; Published online: 5 April 2021

\begin{abstract}
In this paper, introduce a neutrosophicopen sets in neutrosophic topological spaces. Also, discuss about near open sets, their properties and examplesZ-open set which is a union of neutrosophic P-open sets and neutrosophic $\delta$ of a neutrosophicS Z-open set. Moreover, we investigate some of their basic properties and examples of neutrosophic Zinterior and $Z$-closure in a neutrosophic topological spaces.
\end{abstract}

Keywords and phrases: neutrosophic Z-open sets, neutrosophic Z-closed sets, $N Z$ int $(K)$ and $N C(K)$. AMS (2000) Subject classification: 03E72, 54A10, 54A40

\section{$1 \quad$ Introduction}

In mathematics, concept of fuzzy set between the intervals was first introduced by Zadeh [16] in discipline of logic and set theory. The general topology has been framework with fuzzy set was undertaken by Chang [4] as fuzzy topological space. In 1983, Atanassov [2] initiated intuitionistic fuzzy set which contains a membership and nonmembership values. Coker [5] created intuitionistic fuzzy set in a topology entitled as intuitionistic fuzzy topological spaces. The concepts of neutrosophy and neutrosophic set was introduced Smarandache [11, 12] at the beginning of $20^{\text {th }}$ century. Salama and Alblowi [8] in 2012, originated neutrosophic set in a neutrosophic topological space. Saha [13] defined $\delta$-open sets in fuzzy topological spaces. In 2008, Ekici [6] introduced the notion of $e$-open sets in a general topology. In 2014, Seenivasan et. al. [10] introduced fuzzy $e$-open sets in a topological space along with fuzzy $e$ continuity. Vadivel et al. [3] studied fuzzy $e$-open sets in intuitionistic fuzzy topological space. Vadivel et al. [14] introduced $e$-open sets in a neutrosophic topological space. From 2011, El-Maghrabi and Mubarki [7] introduced and studied some properties of Z-open sets and maps in topological spaces. In this paper, we develop the concept of neutrosophic Z-open sets in a neutrosophic topological spaces and also specialized some of their basic properties with examples. Also, we discuss about neutrosophic $Z$-interior and $Z$-closure in neutrosophic topological spaces.

\section{Preliminaries}

The needful basic definitions \& properties of neutrosophic topological spaces are discussed in this section.

Definition 2.1 [9] Let $X$ be a non-empty set. A neutrosophic set (briefly, $N s$ ) $L$ is an object having the form $L=$ $\left\{\left\langle y, \mu_{L}(y), \sigma_{L}(y), v_{L}(y)\right\rangle: y \in X\right\}$ where $\mu_{L} \rightarrow[0,1]$ denote the degree of membership function, $\sigma_{L} \rightarrow[0,1]$ denote the degree of indeterminacy function and $v_{L} \rightarrow[0,1]$ denote the degree of non-membership function respectively of each element $y \in X$ to the set $L$ and $0 \leq \mu_{L}(y)+\sigma_{L}(y)+v_{L}(y) \leq 3$ for each $y \in X$.

Remark 2.1 [9] A $N s L=\left\{\left\langle y, \mu_{L}(y), \sigma_{L}(y), v_{L}(y)\right\rangle: y \in X\right\}$ can be identified to an ordered triple $\left\langle y, \mu_{L}(y), \sigma_{L}(y), v_{L}(y)\right\rangle$ in $[0,1]$ on $X$.

Definition 2.2 [9] Let $X$ be a non-empty set \& the Ns's $L \& M$ in the form $L=\left\{\left\langle y, \mu_{L}(y), \sigma_{L}(y), v_{L}(y)\right\rangle: y \in X\right\}, M=$ $\left\{\left\langle y, \mu_{M}(y), \sigma_{M}(y), v_{M}(y)\right\rangle: y \in X\right\}$, then

(i) $0_{N}=\langle y, 0,0,1\rangle$ and $1_{N}=\langle y, 1,1,0\rangle$,

(ii) $L \subseteq M$ iff $\mu_{L}(y) \leq \mu_{M}(y), \sigma_{L}(y) \leq \sigma_{M}(y) \& v_{L}(y) \geq v_{M}(y): y \in X$,

(iii) $L=M$ iff $L \subseteq M$ and $M \subseteq L$,

(iv) $1_{N}-L=\left\{\left\langle y, v_{L}(y), 1-\sigma_{L}(y), \mu_{L}(y)\right\rangle: y \in X\right\}=L^{c}$,

(v) $L \cup M=\left\{\left\langle y, \max \left(\mu_{L}(y), \mu_{M}(y)\right), \max \left(\sigma_{L}(y), \sigma_{M}(y)\right), \min \left(v_{L}(y), v_{M}(y)\right)\right\rangle: y \in X\right\}$,

(vi) $L \cap M=\left\{\left\langle y, \min \left(\mu_{L}(y), \mu_{M}(y)\right), \min \left(\sigma_{L}(y), \sigma_{M}(y)\right), \max \left(v_{L}(y), v_{M}(y)\right)\right\rangle: y \in X\right\}$.

Definition 2.3 [8] A neutrosophic topology (briefly, $N t$ ) on a non-empty set $X$ is a family $\tau_{N}$ of neutrosophic subsets of $X$ satisfying

(i) $0_{N}, 1_{N} \in \tau_{N}$.

(ii) $L_{1} \cap L_{2} \in \tau_{N}$ for any $L_{1}, L_{2} \in \tau_{N}$.

(iii) $\cup L_{a} \in \tau_{N}, \forall L_{a}: a \in A \subseteq \tau_{N}$.

\footnotetext{
${ }^{1}$ toyesmohan@gmail.com

†avmaths@gmail.com

2 tamil_au@yahoo.com ${ }^{1}$ Department of Mathematics, Government Arts College (Hutonomous), Karur, Tamil Nadu-639 005; Department of Mathematics, Annamalai University, Annamalainagar, Tamil Nadu-608 002 2Department of Mathematics, Thiruvalluvar Goverment Arts College, Rasipuram, Tamil Nadu-637 401 and ${ }^{3}$ Mathematics Section (FEAT), Annamalai University, Annamalainagar, Tamil Nadu-608 002.
} 
Then $\left(X, \tau_{N}\right)$ is called a neutrosophic topological space (briefly, Nts) in $X$. The $\tau_{N}$ elements are called neutrosophic open sets (briefly, Nos) in X. A Ns $C$ is called a neutrosophic closed sets (briefly, $N c s$ ) iff its complement $C^{c}$ is Nos.

Definition 2.4 [8] Let $\left(X, \tau_{N}\right)$ be Nts on $X$ and $L$ be an $N s$ on $X$, then the neutrosophic interior of $L($ briefly, Nint $(L))$ and the neutrosophic closure of $L$ (briefly, $\operatorname{Ncl}(L)$ ) are defined as

$\operatorname{Nint}(L)=\cup\{I: I \subseteq L \& I$ is a $\operatorname{Nos}$ in $X\} N \operatorname{Ncl}(L)=\cap\{I: L \subseteq I \& I$ is a Ncs in $X\}$.

Definition 2.5 [1] Let $\left(X, \tau_{N}\right)$ be $N t s$ on $X$ and $L$ be an $N s$ on $X$. Then $L$ is said to be a neutrosophic regular (resp. pre,

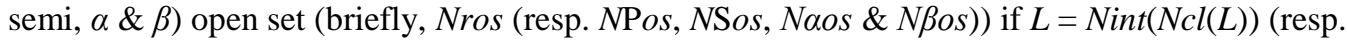

$L \subseteq \operatorname{Nint}(N c l(L)), L \subseteq N c l(\operatorname{Nint}(L)), L \subseteq \operatorname{Nint}(N c l(\operatorname{Nint}(L))) \& L \subseteq N \operatorname{Nol}(\operatorname{Nint}(N c l(L))))$.

The complement of an NPos (resp. NSos, Naos, Nros \& NBos) is called a neutrosophic pre (resp. semi, $\alpha$, regular

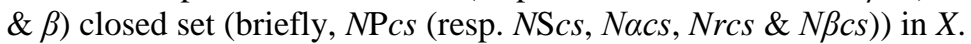

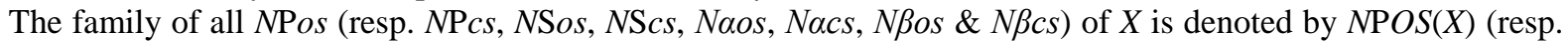
$N \mathrm{PCS}(X), N \mathrm{~S} O S(X), N S C S(X), N \alpha O S(X), N \alpha C S(X), N \beta O S(X) \& N \beta C S(X))$.

Definition 2.6 [14] A set $L$ is said to be a neutrosophic

(i) $\delta$ interior of $L$ (briefly, $N \operatorname{sint}(L)$ ) is defined by $N \operatorname{sint}(L)=\cap \cup\{I: \subseteq I \subseteq L \& I$ is a $N \operatorname{ros}$ in $X\}\}$.

(ii) $\delta$ closure of $L$ (briefly, $N \delta c l(L)$ ) is defined by $N \delta c l(L)=\{A: L \quad A \& A$ is a $N r c s$ in $X$.

Definition 2.7 [14] A set $L$ is said to be a neutrosophic

1. $\delta$-open set (briefly, $N \delta o s$ ) if $L=N \delta \operatorname{int}(L)$.

2. $\delta$-semi open set (briefly, $N \delta \operatorname{Sos})$ if $L \subseteq N c l(N \delta i n t(L))$.

The complement of an $N \delta o s$ (resp. $N \delta$ Sos ) is called a neutrosophic $\delta$ (resp. $\delta$-semi) closed set (briefly, $N \delta c s$ (resp. $N \delta \mathrm{S} c s))$ in $X$.

The family of all $N \delta \mathrm{S} o s$ (resp. $N \delta \mathrm{S} c s$ ) of $X$ is denoted by $N \delta \mathrm{S} O S(X)$ (resp. $N \delta \mathrm{S} C S(X)$ ).

Definition 2.8 [14] A set $K$ is said to be a neutrosophic

(i) $e$-open set (briefly, Neos) if $K \subseteq N \operatorname{Ncl}(N \operatorname{sint}(K)) \cup \operatorname{Nint}(N \delta c l(K))$.

(ii) $e$-closed set (briefly, Necs) if $K \supseteq \operatorname{Ncl}(N \delta \operatorname{int}(K)) \cap \operatorname{Nint}(N \delta c l(K))$.

The complement of a Neos is called a Necs.

The family of all Neos (resp. Necs) of $X$ is denoted by $\mathrm{NeOS}(X)$ (resp. $\mathrm{NeCS}(X)$ ).

\section{$3 \quad$ Neutrosophic $Z$-open sets in Nts}

Throughout the sections $3 \& 4$, let $\left(X, \tau_{N}\right)$ be any Nts. Let $K$ and $M$ be a Ns's in Nts.

Definition 3.1 A set $K$ is said to be a neutrosophic

(i) Z-open set (briefly, $N Z o s)$ if $K \subseteq N \operatorname{cl}(N \operatorname{sint}(K)) \cup \operatorname{Nint}(N \operatorname{cl}(K))$.

(ii) Z-closed set (briefly, $N Z c s)$ if $K \supseteq \operatorname{Ncl}(N \operatorname{sint}(K)) \cap \operatorname{Nint}(N \delta c l(K))$.

The complement of a NZos is called a NZcs.

The family of all NZos (resp. NZcs) of $X$ is denoted by $N Z O S(X)$ (resp. $N Z C S(X)$ ).

Definition 3.2 A set $K$ is said to be a neutrosophic

(i) $Z$ interior of $K$ (briefly, $N \operatorname{Zint}(K)$ ) is defined by $\operatorname{ZZint}(K)=\cap \cup\{A: \subseteq A \subseteq K \& A$ is a $N Z o s$ in $\} X\}$.

(ii) $Z$ closure of $K$ (briefly, $N Z c l(K)$ ) is defined by $N Z c l(K)=\{A: K \quad A \& A$ is a $N Z c s$ in $X$.

Proposition 3.1 The statements are hold but the converse does not true.

(i) Every $N \delta o s$ (resp. $N \delta c s$ ) is a Nos (resp. Ncs).

(ii) Every $N o s$ (resp. $N c s$ ) is a $N \delta \mathrm{S} o s($ resp. $N \delta \mathrm{S} c s$ ).

(iii) Every Nos (resp. Ncs) is a $N \mathrm{P} o s$ (resp. NPcs).

(iv) Every $N \delta \mathrm{S} o s$ (resp. $N \delta \mathrm{S} c s$ ) is a $N Z o s$ (resp. $N Z c s$ ).

(v) Every $N \mathrm{P} o s$ (resp. $N \mathrm{P} c s$ ) is a $N Z o s$ (resp. $N Z c s$ ).

(vi) Every NZos (resp. NZcs) is a Neos (resp. Necs).

Proof. The proof of (i), (ii) \& (iii) are studied in $[14,15]$.

(iv) $K$ is a $N \delta$ Sos, then $K \subseteq N \operatorname{cl}(N \operatorname{dint}(K)) \subseteq N \operatorname{cl}(N \operatorname{dint}(K)) \cup \operatorname{Nint}(N c l(K)) . \therefore K$ is a $N Z o s$.

(v) $K$ is a $N$ Pos, then $K \subseteq \operatorname{Nint}(N c l(K)) \subseteq N c l(N \delta i n t(K)) \cup \operatorname{Nint}(N c l(K)) . \therefore K$ is a $N Z o s$.

(vi) $K$ is a $N Z o s$ then $K \subseteq N \operatorname{Nol}(N \operatorname{dint}(K)) \cup \operatorname{Nint}(N c l(K))$. So $K \subseteq N \operatorname{cl}(N \operatorname{dint}(K)) \cup \operatorname{Nint}(N c l(K)) \subseteq N c l(N \operatorname{dint}(K)) \cup$ $\operatorname{Nint}(N \delta c l(K)) . \therefore K$ is a Neos.

It is also true for their respective closed sets.

Remark 3.1 The diagram shows NZos's in fnts. 


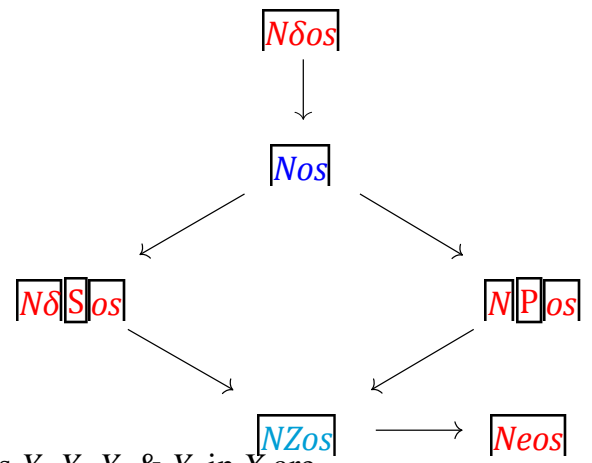

Example 3.1 Let $Y=\{a, b, c\}$ and define $N$ 's $Y_{1}, Y_{2}, Y_{3} \& Y_{4}$ in $X$ are

$$
\begin{aligned}
Y_{1} & =\left\langle Y,\left(\frac{\mu_{a}}{0.2}, \frac{\mu_{b}}{0.3}, \frac{\mu_{c}}{0.4}\right),\left(\frac{\sigma_{a}}{0.5}, \frac{\sigma_{b}}{0.5}, \frac{\sigma_{c}}{0.5}\right),\left(\frac{\nu_{a}}{0.8}, \frac{\nu_{b}}{0.7}, \frac{\nu_{c}}{0.6}\right)\right\rangle, \\
Y_{2} & =\left\langle Y,\left(\frac{\mu_{a}}{0.1}, \frac{\mu_{b}}{0.1}, \frac{\mu_{c}}{0.4}\right),\left(\frac{\sigma_{a}}{0.5}, \frac{\sigma_{b}}{0.5}, \frac{\sigma_{c}}{0.5}\right),\left(\frac{\nu_{a}}{0.9}, \frac{\nu_{b}}{0.9}, \frac{\nu_{c}}{0.6}\right)\right\rangle \\
Y_{3} & =\left\langle Y,\left(\frac{\mu_{a}}{0.2}, \frac{\mu_{b}}{0.2}, \frac{\mu_{c}}{0.3}\right),\left(\frac{\sigma_{a}}{0.5}, \frac{\sigma_{b}}{0.5}, \frac{\sigma_{c}}{0.5}\right),\left(\frac{\nu_{a}}{0.8}, \frac{\nu_{b}}{0.8}, \frac{\nu_{c}}{0.7}\right)\right\rangle, \\
Y_{4} & =\left\langle Y,\left(\frac{\mu_{a}}{0.2}, \frac{\mu_{b}}{0.4}, \frac{\mu_{c}}{0.4}\right),\left(\frac{\sigma_{a}}{0.5}, \frac{\sigma_{b}}{0.5}, \frac{\sigma_{c}}{0.5}\right),\left(\frac{\nu_{a}}{0.8}, \frac{\nu_{b}}{0.6}, \frac{\nu_{c}}{0.6}\right)\right\rangle \\
Y_{5} & =\left\langle Y,\left(\frac{\mu_{a}}{0.8}, \frac{\mu_{b}}{0.7}, \frac{\mu_{c}}{0.8}\right),\left(\frac{\sigma_{a}}{0.5}, \frac{\sigma_{b}}{0.5}, \frac{\sigma_{c}}{0.5}\right),\left(\frac{\nu_{a}}{0.2}, \frac{\nu_{b}}{0.3}, \frac{\nu_{c}}{0.2}\right)\right\rangle,
\end{aligned}
$$

Then we have $\tau_{N}=\left\{0_{N}, Y_{1}, Y_{2}, 1_{N}\right\}$ is a Nts in $X$, then

(i) $Y_{3}$ is a NPos but not Nos.

(ii) $Y_{4}$ is a NZos but not NPos.

(iii) $Y_{5}$ is a Neos but not NZos.

Example 3.2 Let $Y=\{a, b, c\}$ and define $N s^{\prime} s Y_{1}, Y_{2} \& Y_{3}$ in $X$ are

$$
\begin{aligned}
& Y_{1}=\left\langle Y,\left(\frac{\mu_{a}}{0.4}, \frac{\mu_{b}}{0.6}, \frac{\mu_{c}}{0.5}\right),\left(\frac{\sigma_{a}}{0.5}, \frac{\sigma_{b}}{0.5}, \frac{\sigma_{c}}{0.5}\right),\left(\frac{\nu_{a}}{0.6}, \frac{\nu_{b}}{0.4}, \frac{\nu_{c}}{0.5}\right)\right\rangle \\
& Y_{2}=\left\langle Y,\left(\frac{\mu_{a}}{0.6}, \frac{\mu_{b}}{0.4}, \frac{\mu_{c}}{0.4}\right),\left(\frac{\sigma_{a}}{0.5}, \frac{\sigma_{b}}{0.5}, \frac{\sigma_{c}}{0.5}\right),\left(\frac{\nu_{a}}{0.4}, \frac{\nu_{b}}{0.6}, \frac{\nu_{c}}{0.6}\right)\right\rangle, \\
& Y_{3}=\left\langle Y,\left(\frac{\mu_{a}}{0.4}, \frac{\mu_{b}}{0.5}, \frac{\mu_{c}}{0.5}\right),\left(\frac{\sigma_{a}}{0.5}, \frac{\sigma_{b}}{0.5}, \frac{\sigma_{c}}{0.5}\right),\left(\frac{\nu_{a}}{0.6}, \frac{\nu_{b}}{0.5}, \frac{\nu_{c}}{0.5}\right)\right\rangle .
\end{aligned}
$$

Then we have $\tau_{N}=\left\{0_{N}, Y_{1}, Y_{2}, Y_{1} \cup Y_{2}, Y_{1} \cap Y_{2}, 1_{N}\right\}$ is a Nts in $X$, then $Y_{3}$ is a NZos but not ${ }^{N \delta}$ Sos.

The other implications are shown in [14].

Theorem 3.1 Let $\left(X, \tau_{N}\right)$ be a Nts. Then if $M \in N \delta O S(X)$ and $M \in N Z O S(X)$, then $H \cap M$ is NZo.

Proof. Suppose that $H \in N \delta O S(X)$. Then $H=\operatorname{Nint}_{\delta}(H)$. Since $M \in N Z O S(X)$, then $M \subseteq N c l\left(\operatorname{Nint}_{\delta}(M)\right) \cup \operatorname{Nint}(\operatorname{Ncl}(M))$ and hence

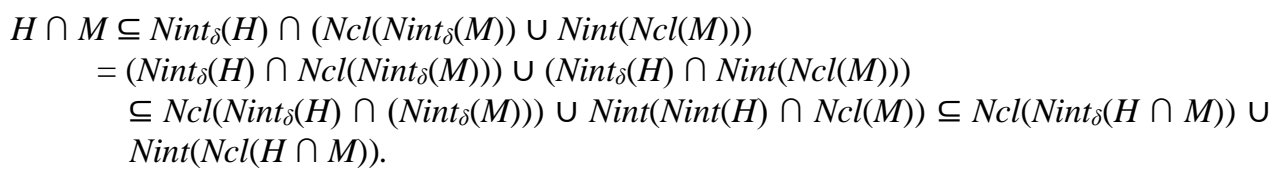

Thus $H \cap M \subseteq N c l\left(\operatorname{Nint}_{\delta}(H \cap M)\right) \cup \operatorname{Nint}(N c l(H \cap M))$. Therefore, $H \cap M$ is $N Z o$.

Proposition 3.2 Let $\left(X, \tau_{N}\right)$ be a Nts. Then the closure of a NZo set of $X$ is NSo.

Proof. Let $H \in N Z O S(X)$. Then

$$
\begin{aligned}
\operatorname{Ncl}(H) & \subseteq \operatorname{Ncl}\left(N c l\left(\operatorname{Nint}_{\delta}(H)\right) \cup \operatorname{Nint}(N c l(H))\right) \\
& \subseteq \operatorname{Ncl}\left(\operatorname{Nint}_{\delta}(H)\right) \cup \operatorname{Ncl}(\operatorname{Nint}(N c l(H)))=\operatorname{Ncl}(\operatorname{Nint}(N c l(H))) .
\end{aligned}
$$

Therefore, $\mathrm{Ncl}(H)$ is $N \mathrm{~S} o$.

Theorem 3.2 The statements are true.

(i) $\operatorname{NPcl}(K) \supseteq K \cup \operatorname{Ncl}(\operatorname{Nint}(K))$.

(ii) $\operatorname{NPint}(K) \subseteq K \cap \operatorname{Nint}(\operatorname{Ncl}(K))$.

(iii) $N \delta \mathrm{S} c l(K) \supseteq K \cup \operatorname{Nint}(N \delta c l(K))$.

(iv) $N \delta \operatorname{Sint}(K) \subseteq K \cap N c l(N \delta \operatorname{sint}(K))$.

Proof. (i) Since $N \mathrm{P} c l(K)$ is $N \mathrm{P} c s$, we have

Thus $K \cup N c l(\operatorname{Nint}(K)) \subseteq N \mathrm{P} c l(K)$.

$N \operatorname{Nol}(\operatorname{Nint}(K)) \subseteq \operatorname{Ncl}(\operatorname{Nint}(N \mathrm{Pcl}(K))) \subseteq \operatorname{NPcl}(K)$. 
The other cases are similar.

Theorem 3.3 Let $K$ is a NZos iff $K=N \operatorname{Pint}(K) \cup N \delta \operatorname{Sint}(K)$.

Proof. Let $K$ is a NZos. Then $K \subseteq N \operatorname{Nol}(N \operatorname{int}(K)) \cup \operatorname{Nint}(N c l(K))$. By Theorem 3.2, we have

$$
\begin{gathered}
N \operatorname{Pint}(K) \cup N \delta \operatorname{Sint}(K)=K \cap(N i n t(N c l(K))) \cup(K \cap N \operatorname{Ncl}(N \operatorname{dint}(K)))=K \cap(\operatorname{Nint}(N c l(K))) \cup \\
N \operatorname{Ncl}(N \operatorname{dint}(K))=K .
\end{gathered}
$$

Conversely, if $K=N \operatorname{Pint}(K) \cup N \delta \operatorname{Sint}(K)$ then, by Theorem 3.2

$$
\begin{aligned}
K & =N \operatorname{Pint}(K) \cup N \delta \operatorname{Sint}(K) \\
& =(K \cap \operatorname{Nint}(N \operatorname{cl}(K))) \cup(K \cap N \operatorname{Ncl}(N \operatorname{iint}(K))) \\
& =K \cap(\operatorname{Nint}(N \operatorname{cl}(K)) \cup N \operatorname{Nol}(N \operatorname{int}(K))) \subseteq \operatorname{Nint}(N \operatorname{cl}(K)) \cup \operatorname{Ncl}(N \operatorname{dint}(K))
\end{aligned}
$$

and hence $K$ is a NZos.

Theorem 3.4 The union (resp. intersection) of any family of $N Z O S(X)$ (resp. $N Z C S(X))$ is a $N Z O S(X)($ resp. $\operatorname{NZCS}(X))$.

Proof. Let $\left\{K_{a}: a \in \tau_{N}\right\}$ be a family of NZos's. For each $a \in \tau_{N}, K_{a} \subseteq \operatorname{Ncl}\left(\operatorname{Nint}\left(K_{a}\right)\right) \cup \operatorname{Nint}\left(\operatorname{Ncl}\left(K_{a}\right)\right)$.

The other case is similar.

$$
\begin{aligned}
\bigcup_{a \in \tau_{N}} K_{a} & \subseteq \bigcup_{a \in \tau_{N}} \operatorname{Ncl}\left(\operatorname{Nint}\left(K_{a}\right)\right) \cup \operatorname{Nint}\left(\operatorname{Ncl}\left(K_{a}\right)\right) \\
& \subseteq \operatorname{Ncl}\left(\operatorname{Nint}\left(\cup K_{a}\right)\right) \cup \operatorname{Nint}\left(N c l\left(\cup K_{a}\right)\right)
\end{aligned}
$$

Remark 3.2 The intersection of two NZos's need not be NZos.

Example 3.3 Let $Y=\{a, b\}$ and define $N s$ 's $Y_{1}, Y_{2} \& Y_{3}$ in $X$ are

$$
\begin{aligned}
& Y_{1}=\left\langle Y,\left(\frac{\mu_{a}}{0.2}, \frac{\mu_{b}}{0.1}\right),\left(\frac{\sigma_{a}}{0.5}, \frac{\sigma_{b}}{0.5}\right),\left(\frac{\nu_{a}}{0.7}, \frac{\nu_{b}}{0.5}\right)\right\rangle \\
& Y_{2}=\left\langle Y,\left(\frac{\mu_{a}}{0.3}, \frac{\mu_{b}}{0.5}\right),\left(\frac{\sigma_{a}}{0.5}, \frac{\sigma_{b}}{0.5}\right),\left(\frac{\nu_{a}}{0.7}, \frac{\nu_{b}}{0.2}\right)\right\rangle, \\
& Y_{3}=\left\langle Y,\left(\frac{\mu_{a}}{0.1}, \frac{\mu_{b}}{0.2}\right),\left(\frac{\sigma_{a}}{0.5}, \frac{\sigma_{b}}{0.5}\right),\left(\frac{\nu_{a}}{0.1}, \frac{\nu_{b}}{0.1}\right)\right\rangle .
\end{aligned}
$$

Then we have $\tau_{N}=\left\{0_{N}, Y_{1}, 1_{N}\right\}$ is a Nts in $X$, then $Y_{2} \& Y_{3}$ are NZos but $Y_{2} \cap Y_{3}$ is not NZos.

Proposition 3.3 Let $K$ is a

(i) $N Z o s$ and $N \operatorname{dint}(K)=0_{N}$, then $K$ is a $N \mathrm{P} o s$.

(ii) $N Z o s$ and $N \operatorname{cl}(K)=0_{N}$, then $K$ is a $N \delta$ Sos.

(iii) $N Z o s$ and $N \delta c s$, then $K$ is a $N \delta$ Sos.

(iv) $N \delta$ Sos and $N c s$, then $K$ is a NZos.

Proof. (i) Let $K$ be a NZos, that is

$$
K \subseteq N \operatorname{cl}(N \operatorname{sint}(K)) \cup \operatorname{Nint}(N c l(K))=0_{N} \cup \operatorname{Nint}(N c l(K))=\operatorname{Nint}(N c l(K))
$$

Hence $K$ is a NPos.

(ii) Let $K$ be a NZos, that is

$$
K \subseteq N \operatorname{cl}(N \operatorname{sint}(K)) \cup \operatorname{Nint}(N \operatorname{cl}(K))=N \operatorname{cl}(N \operatorname{sint}(K)) \cup 0_{N}=N \operatorname{cl}(N \operatorname{sint}(K))
$$

Hence $K$ is a $N \delta$ Sos.

(iii) Let $K$ be a $N Z o s$ and $N \delta c s$, that is

Hence $K$ is a $N \delta$ Sos.

$$
K \subseteq N \operatorname{Nol}(N \operatorname{int}(K)) \cup \operatorname{Nint}(N \operatorname{cl}(K))=N \operatorname{cl}(N \operatorname{sint}(K)) \cup \operatorname{Nint}(N c l(K))=N \operatorname{cl}(N \operatorname{int}(K)) .
$$

(iv) Let $K$ be a $N \delta$ Sos and $N c s$, that is

$$
K \subseteq N \operatorname{cl}(N \operatorname{iint}(K)) \subseteq N \operatorname{cl}(N \operatorname{iint}(K)) \cup \operatorname{Nint}(N \operatorname{cl}(K))
$$

Hence $K$ is a NZos.

Theorem 3.5 Let $K$ be a $N Z c s$ (resp. NZos) iff $K=N Z c l(K)(\operatorname{resp} . K=N Z i n t(K))$.

Proof. Suppose $K=N Z c l(K)=\cap\{A: K \subseteq A \& A$ is a $N Z c s\}$. This means $K \in \cap\{A: K \subseteq A \& A$ is a NZcs $\}$ and hence $K$ is $N Z c s$.

Conversely, suppose $K$ be a $N Z c s$ in $X$. Then, we have $K \in \cap\{A: K \subseteq A \& A$ is a $N Z c s\}$. Hence, $K \subseteq A$ implies $K$ $=\bigcap\{A: K \subseteq A \& A$ is a $N Z c s\}=N Z c l(K)$.

Similarly for $K=N Z \operatorname{int}(K)$.

Proposition 3.4 Let $K$ and $L$ are in $X$, then

(i) $\operatorname{NZcl}(\overline{K)}=N \overline{\operatorname{int}(K), N Z i n t}(K)=N \overline{Z c l}(K)$.

(ii) $\operatorname{NZcl}(K \cup L) \supseteq N Z c l(K) \cup N Z c l(L), N Z c l(K \cap L) \subseteq N Z c l(K) \cap N Z c l(L)$.

(iii) $\operatorname{NZint}(K \cup L) \supseteq N Z \operatorname{Zint}(K) \cup N Z \operatorname{int}(L), N Z i \operatorname{int}(K \cap L) \subseteq N Z \operatorname{Rint}(K) \cap N Z \operatorname{int}(L)$.

Proof.

(i) The proof is directly from definition. 
(ii) $K \subseteq K \cup L$ or $L \subseteq K \cup L$. Hence $N Z c l(K) \subseteq N Z c l(K \cup L)$ or $N Z c l(L) \subseteq N Z c l(K \cup L)$. Therefore, $N Z c l(K \cup L) \supseteq$ $N Z c l(K) \cup N Z c l(L)$. The other one is similar.

(iii) $K \subseteq K \cup L$ or $L \subseteq K \cup L$. Hence $\operatorname{NZint}(K) \subseteq \operatorname{NZint}(K \cup L)$ or $\operatorname{NZint}(L) \subseteq \operatorname{NZint}(K \cup L)$. Therefore, $\operatorname{NZint}(K \cup L) \supseteq \operatorname{NZint}(K) \cup \operatorname{NZint}(L)$. The other one is similar.

$\Xi$

Remark 3.3 The equality of (ii) in Proposition 3.4 can not be true in the given example.

Example 3.4 Let $Y=\{a, b, c, d\}$ and define $N s$ 's $Y_{1}, Y_{2}, Y_{3} \& Y_{4}$ in $X$ are

$$
\begin{gathered}
Y_{1}=\left\langle Y,\left(\frac{\mu_{a}}{1}, \frac{\mu_{b}}{0}, \frac{\mu_{c}}{0.2}, \frac{\mu_{d}}{0}\right),\left(\frac{\sigma_{a}}{0.5}, \frac{\sigma_{b}}{0.5}, \frac{\sigma_{c}}{0.5}, \frac{\sigma_{d}}{0.5}\right),\left(\frac{\nu_{a}}{0}, \frac{\nu_{b}}{1}, \frac{\nu_{c}}{0.7}, \frac{\nu_{d}}{1}\right)\right\rangle \\
Y_{2}=\left\langle Y,\left(\frac{\mu_{a}}{0}, \frac{\mu_{b}}{1}, \frac{\mu_{c}}{0}, \frac{\mu_{d}}{0}\right),\left(\frac{\sigma_{a}}{0.5}, \frac{\sigma_{b}}{0.5}, \frac{\sigma_{c}}{0.5}, \frac{\sigma_{d}}{0.5}\right),\left(\frac{\nu_{a}}{1}, \frac{\nu_{b}}{0}, \frac{\nu_{c}}{1}, \frac{\nu_{d}}{0.1}\right)\right\rangle, \\
Y_{3}=\left\langle Y,\left(\frac{\mu_{a}}{1}, \frac{\mu_{b}}{0}, \frac{\mu_{c}}{0}, \frac{\mu_{d}}{1}\right),\left(\frac{\sigma_{a}}{0.5}, \frac{\sigma_{b}}{0.5}, \frac{\sigma_{c}}{0.5}, \frac{\sigma_{d}}{0.5}\right),\left(\frac{\nu_{a}}{0}, \frac{\nu_{b}}{0.2}, \frac{\nu_{c}}{0}, \frac{\nu_{d}}{0}\right)\right\rangle, \\
Y_{4}=\left\langle Y,\left(\frac{\mu_{a}}{0}, \frac{\mu_{b}}{0.9}, \frac{\mu_{c}}{0.3}, \frac{\mu_{d}}{1}\right),\left(\frac{\sigma_{a}}{0.5}, \frac{\sigma_{b}}{0.5}, \frac{\sigma_{c}}{0.5}, \frac{\sigma_{d}}{0.5}\right),\left(\frac{\nu_{a}}{1}, \frac{\nu_{b}}{0}, \frac{\nu_{c}}{0.2}, \frac{\nu_{d}}{0}\right)\right\rangle,
\end{gathered}
$$

Then we have $\tau_{N}=\left\{0_{N}, Y_{1}, Y_{2}, Y_{1} \cap Y_{2}, 1_{N}\right\}$ is a $N t s$ in $X$, then $\operatorname{NZcl}\left(Y_{3} \cup Y_{4}\right)=\square \operatorname{NZcl}\left(Y_{3}\right) \cup \operatorname{NZcl}\left(Y_{4}\right)$.

Proposition 3.5 Let $K$ be a neutrosophic set in a neutrosophic topological space $X$. Then $\operatorname{Nint}(K) \subseteq N Z \operatorname{int}(K) \subseteq K \subseteq$ $\operatorname{NZcl}(K) \subseteq N \operatorname{cl}(K)$.

Proof. It follows from the definitions of corresponding operators. $\quad \Xi$ Theorem 3.6 Let $K$ and $L$ in $X$, then the NZint sets have

(i) $\operatorname{NZcl}\left(0_{N}\right)=0_{N}, \operatorname{NZcl}\left(1_{N}\right)=1_{N}$.

(ii) $\operatorname{NZcl}(K)$ is a $N Z c s$ in $X$.

(iii) $\operatorname{NZcl}(K) \subseteq N Z c l(L)$ if $K \subseteq L$.

(iv) $K \subseteq N Z c l(K)$.

(v) $K$ is $N Z c$ set in $X \Leftrightarrow N Z c l(K)=K$.

(vi) $\operatorname{NZint}(N \operatorname{Zint}(K))=\operatorname{NZint}(K)$.

Proof. The proofs (i) to (iv) and (vi) are directly from definitions of $\mathrm{NZcl}$ set.

(v) Let $K$ be $N Z c$ set in $X$. By using Proposition $\overline{3} .4, K$ is NZo set in $X$. By Proposition 3.4, NZint $(\bar{K})=\bar{K} \Leftrightarrow$

$$
\operatorname{NZcl}(K)=K \Leftrightarrow \operatorname{NZcl}(K)=K \text {. }
$$

Theorem 3.7 Let $K$ and $L$ in $X$, then the NZint sets have

(i) $\operatorname{NZint}\left(0_{N}\right)=0_{N}, \operatorname{NZint}\left(1_{N}\right)=1_{N}$.

(ii) $\operatorname{NZint}(K)$ is a $N Z o s$ in $X$.

(iii) $\operatorname{NZint}(K) \subseteq N \operatorname{Zint}(L)$ if $K \subseteq L$.

(iv) $\operatorname{NZint}(N \operatorname{Zint}(K))=\operatorname{NZint}(K)$.

Proof. The proofs are directly from definitions of NZint set.

Proposition 3.6 If $K$ and $L$ is in $X$, then (i) $N Z c l(K) \supseteq K \cup N Z c l(N Z i n t(K))$.

(ii) $\operatorname{NZint}(K) \subseteq K \cap \operatorname{Nint}(\operatorname{NZcl}(K))$.

(iii) $\operatorname{Nint}(N Z c l(K)) \supseteq \operatorname{Nint}(N Z c l(N Z i n t(K)))$.

Proof. (i) By Theorem 3.6 $K \subseteq N Z c l(K) \rightarrow(1)$. Again using Theorem 3.6, $N \operatorname{Zint}(K) \subseteq K$. Then $N Z c l(N Z i n t(K)) \subseteq$ $N Z c l(K) \rightarrow(2)$. By (1) and (2) we have, $K \cup N Z c l(N Z i n t(K)) \subseteq N Z c l(K)$.

(ii) By Theorem 3.6, NZint $(K) \subseteq K \rightarrow(1)$. Again using Theorem 3.6, $K \subseteq N \operatorname{Nint}(K)$. Then $N Z \operatorname{Zint}(K) \subseteq$ $\operatorname{Nint}(N Z c l(K) \rightarrow(2)$. By (1) and (2) we have, $N \operatorname{Zint}(K) \subseteq K \cup \operatorname{Nint}(N Z c l(K))$.

(iii) By Theorem 3.6, $N Z c l(K) \subseteq N c l(K)$, we get $\operatorname{Nint}(N Z c l(K)) \subseteq N i n t(N c l(K))$. Hence (iii).

(iv) By (i), $N Z c l(K) \supseteq K \cup N Z(N Z \operatorname{Zint}(K))$. We have, $\operatorname{Nint}(N Z c l(K)) \supseteq \operatorname{Nint}(K \cup N Z c l(N Z i n t(K)))$. Since $\operatorname{Nint}(K$ $\cup L) \supseteq \operatorname{Nint}(K) \cup \operatorname{Nint}(L), \operatorname{Nint}(N Z c l(K)) \supseteq \operatorname{Nint}(K) \cup \operatorname{Nint}(N Z c l(N Z i n t(K))) \supseteq \operatorname{Nint}(N Z c l(N Z \operatorname{int}(K))) . \Xi$

(v)

\section{Conclusion}

We have studied about neutrosophic Z-open set and neutrosophic Z-closed set and their respective interior and closure operators of neutrosophic topological space in this paper. Also studied some of their fundamental properties along with examples in Nts. Also, we have discussed a near open sets of neutrosophic Z-open sets in Nts. In future, we can be extended to neutrosophic $Z$ continuous mappings, neutrosophic $Z$-open mappings and neutrosophic $Z$-closed mappings in Nts. 


\section{References}

1. I. Arokiarani, R. Dhavaseelan, S. Jafari and M. Parimala, On some new notions and functions in neutrosophic topological spaces, Neutrosophic Sets and Systems, 16 (2017), 16-19.

2. K. Atanassov, Intuitionistic fuzzy sets, Fuzzy Sets and Systems, 20 (1986), 87-96.

3. V. Chandrasekar, D. Sobana and A. Vadivel, On Fuzzy e-open Sets, Fuzzy e-continuity and Fuzzy ecompactness in Intuitionistic Fuzzy Topological Spaces, Sahand Communications in Mathematical Analysis (SCMA), 12 (1) (2018), 131-153.

4. C. L. Chang, Fuzzy topological spaces, J. Math. Anal. Appl., 24 (1968), 182-190.

5. D. Coker, An introduction to intuitionistic fuzzy topological spaces, Fuzzy sets and systems, 88 (1997), 8189.

6. Erdal Ekici, On e-open sets, $\mathrm{DP}^{\star}$-sets and $\mathrm{DP} \epsilon^{*}$-sets and decomposition of continuity, The Arabian Journal for Science and Engineering, 33 (2A) (2008), 269-282.

7. A. I. El-Magharabi and A. M. Mubarki, Z-open sets and Z-continuity in topological spaces, International Journal of Mathematical Archive, 2 (10) (2011), 1819-1827.

8. A. A. Salama and S. A. Alblowi, Neutrosophic set and neutrosophic topological spaces, IOSR Journal of Mathematics, 3 (4) (2012), 31-35.

9. A. A. Salama and F. Smarandache, Neutrosophic crisp set theory, Educational Publisher, Columbus, Ohio, USA, 2015.

10. V. Seenivasan and K. Kamala, Fuzzy e-continuity and fuzzy e-open sets, Annals of Fuzzy Mathematics and Informatics, 8 (2014), 141-148.

11. F. Smarandache, A Unifying field in logics: neutrosophic logic. neutrosophy, neutrosophic set, neutrosophic probability, American Research Press, Rehoboth, NM, (1999).

12. F. Smarandache, Neutrosophy and neutrosophic logic, First International Conference on Neutrosophy, Neutrosophic Logic, Set, Probability, and Statistics, University of New Mexico, Gallup, NM 87301, USA (2002).

13. Supriti Saha, Fuzzy $\delta$-continuous mappings, Journal of Mathematical Analysis and Applications, 126 (1987), 130-142. [14] A. Vadivel, C. John Sundar and P. Thangaraja, Neutrosophic e-open sets in a topological spaces, Submitted.

14. V. Venkateswara Rao and Y. Srinivasa Rao, Neutrosophic pre-open sets and pre-closed sets in Neutrosophic topology, International Journal of Chem Tech Research, 10 (10) (2017), 449-458.

15. L. A. Zadeh, Fuzzy sets, Information and control, 8 (1965), 338-353. 